\title{
Pion Correlations and Two-Particle Bound States
}

\author{
M.Shmatikov \\ Institute for Theoretical Physics II, \\ Ruhr Universität Bochum, D-44780 Bochum, Germany \\ and \\ Russian Research Center "Kurchatov Institute", \\ 123182 Moscow, Russia
}

\begin{abstract}
A method to investigate possible loosely bound dibaryon states is suggested. The momentum dependence of a two-pion correlation function is shown to depend on the (non)existence of a bound state.
\end{abstract}

The only dibaryon state which existence does not cause at present any doubts is the deuteron. There is also a plausible candidate suggested for the explanation of the forward angle cross section of the pionic double charge exchange [1]. It is unnecessary to stress that its experimental observation became possible because of the extremely small width of the resonance: $\Gamma \lesssim 0.5 \mathrm{MeV}$ [1].

Other dibaryon states with much larger width, exemplified by a $\Delta \Delta$ bound state [2], are also conceivable. A naive estimate of its width $\Gamma \gtrsim 2 \Gamma_{\Delta} \approx$ $300 \mathrm{MeV}$ prompts, however, that it will hardly manifest itself as a resonance peak.

Here we address the problem of observing possible bound states of twobaryon resonances. To be more specific we consider a pair of two baryon resonances $\left(B_{1}\right.$ and $B_{2}$ ), their interaction being strong enough to produce a (loosely-) bound state. Were the bound-state components stable it would, like the deuteron, exist for an infinitely long time. However, because of the baryons' decay the bound state itself exists for a finite time. The only signal of a bound state emergence will be then the localization of the $B_{1}$ and $B_{2}$ particles in some finite spatial region.

A dominant decay channel of a baryon resonance is usually that with the

*Permanent address 
$\pi$-meson emission:

$$
B \rightarrow B^{\prime}+\pi
$$

Then the $\left(B_{1} B_{2}\right)$ bound state will decay with the emission of two pions:

$$
\left(B_{1} B_{2}\right) \rightarrow B_{1}^{\prime}+B^{\prime}{ }_{2}+\pi+\pi
$$

In the case of a $(\Delta \Delta)$ bound state the decay pattern (11) translates into

$$
(\Delta \Delta) \rightarrow N+N+\pi+\pi
$$

Hereafter we shall address specifically the process (2) though all the results are applicable to a more general case (11).

Spatial distribution of two $\Delta$-isobars can be scanned using the two-particle correlation in the momentum space. The latter in the form of the pionic interferometry is routinely applied for extracting information about the spacetime structure of the emitting sources in heavy-ion collisions [3].

Forbidding complexity of the multiparticle process (11) (and (2)) necessitates making some simplifying approximations. In the vein of the $N_{c} \rightarrow \infty$

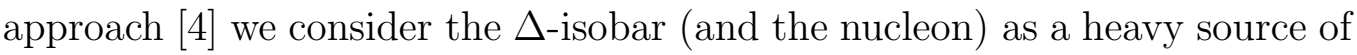
$\pi$-mesons. Then the process (2) can be viewed as an emission of the pions by two heavy sources superimposed by the motion of the sources which is not affected by the pion emission.

Separating the system under consideration into two components: a fast ( $\Delta \rightarrow N \pi$ decay) and a slow (relative motion of two $\Delta$-isobars and nucleons) ones we apply the adiabatic approximation [5]

Consider first two $\Delta$-isobars at a distance $\vec{R}_{\Delta \Delta}$. Correlation between two (identical) pions due to the quantum mechanical uncertainty depends on the sources' separation $\vec{R}_{\Delta \Delta}$. In what follows we use the formalism developed in [6]. Let $\psi_{\pi \pi}$ be the wave function of the $\pi \pi$ system. Its time evolution is governed by the hamiltonian $H$ :

$$
\psi_{\pi \pi}\left(\vec{r}_{a}, \vec{r}_{b} ; t\right)=\exp \left(-i H\left(\vec{r}_{a}, \vec{r}_{b}\right)\left(t-t_{0}\right)\right) \psi_{\pi \pi}\left(\vec{r}_{a}, \vec{r}_{b} ; t_{0}\right)
$$

We define also an asymptotic state with definite values of the $\pi$-meson momenta

$$
\phi_{\vec{p}_{a}, \vec{p}_{b}}\left(\vec{r}_{a}, \vec{r}_{b} ; t\right)=\exp \left(-i\left(E_{a}+E_{b}\right) t\right) \exp \left(i\left(\vec{p}_{a} \vec{r}_{a}+\vec{p}_{b} \vec{r}_{b}\right)\right)
$$


where $E_{a, b}=\sqrt{m_{\pi}^{2}+\vec{p}_{a, b}^{2}}$ are the particle energies. Then the measured twoparticle amplitude equals [6]:

$$
\begin{aligned}
A\left(\vec{p}_{a}, \vec{p}_{b}\right)= & \lim _{t \rightarrow \infty} \int d^{3} r_{a} d^{3} r_{b} \psi_{\pi \pi}\left(\vec{r}_{a}, \vec{r}_{b}, t_{0}\right) \\
& \times\left[\exp \left[-i H\left(\vec{r}_{a}, \vec{r}_{b}\right)\left(t-t_{0}\right)\right] \phi_{\vec{p}_{a}, \vec{p}_{b}}\left(\vec{r}_{a}, \vec{r}_{b} ; t\right)\right]^{*}
\end{aligned}
$$

The values of $t$ for which this expression is valid will be discussed below.

It is convenient to introduce center-of-mass and relative coordinates of two $\pi$-mesons:

$$
\vec{r}=\vec{r}_{a}-\vec{r}_{b} ; \quad \vec{R}=\frac{\vec{r}_{a}+\vec{r}_{b}}{2}
$$

and also the total and the relative momenta of the pair:

$$
\vec{q}=\vec{p}_{a}-\vec{p}_{b} ; \quad \vec{P}=\vec{p}_{a}+\vec{p}_{b}
$$

Using these coordinates the asymptotic wave function can be rewritten as:

$$
\phi_{\vec{p}_{a}, \vec{p}_{b}}\left(\vec{r}_{a}, \vec{r}_{b} ; t\right)=e^{i(\vec{P} \vec{R}-E t)} e^{\frac{\vec{q} \vec{r}}{2}}
$$

where $E=E_{a}+E_{b}$ is the total energy of the $\pi \pi$ pair. The total energy $E$ may be separated then into the c.m.s motion energy $E_{\mathrm{cm}}$ and the relative motion energy $E_{\mathrm{rel}}$. The former obviously reads $E_{\mathrm{cm}}=\sqrt{\vec{P}^{2}+4 m_{\pi}^{2}}$. Then $E_{\mathrm{rel}}=E-E_{\mathrm{cm}}$. In a frame where $\vec{P} \approx 0$ the relative motion energy reduces to $E_{\text {rel }} \approx \vec{q}^{2} /\left(4 m_{\pi}\right)$. We make a simplifying assumption that the emitted $\pi$ mesons do not interact with each other. (The validity of this approximation is discussed below.) Then the two-pion amplitude (5) can be cast in the form [6]:

$$
A\left(\vec{p}_{a}, \vec{p}_{b}\right)=e^{i E t_{0}} \int d^{3} r d^{3} R e^{-i \vec{P} \vec{R}} e^{i \vec{q} \vec{r} / 2} \psi_{\pi \pi}\left(\vec{R}+\frac{\vec{r}}{2}, \vec{R}-\frac{\vec{r}}{2} ; t_{0}\right)
$$

We assume that two pions are emitted in the decays of baryons independently, implying that at some time moment $t_{a}$ the two-particle wave function $\psi_{\pi \pi}$ factorizes:

$$
\psi_{\pi \pi}\left(\vec{r}_{a}, \vec{r}_{b} ; t_{a}\right)=\frac{1}{\sqrt{2}}\left[\psi_{a}\left(\vec{r}_{a} ; t_{a}\right) \psi_{b}\left(\vec{r}_{b} ; t_{a}\right)+\psi_{a}\left(\vec{r}_{b} ; t_{a}\right) \psi_{b}\left(\vec{r}_{a} ; t_{a}\right)\right]
$$

Consider the case when the $\pi$-meson, in the state described by the $\psi_{b}$ wave function, was emitted earlier than that in the $\psi_{a}$ state at some time 
$t_{b}<t_{a}$. Then by the moment $t_{a}$ (when the second pion was emitted) its wave function has acquired some phase:

$$
\psi_{b}\left(\vec{r} ; t_{a}\right)=e^{-i h(\vec{r})\left(t_{a}-t_{b}\right)} \psi_{b}\left(\vec{r} ; t_{b}\right)
$$

where $h$ is the one-particle Hamiltonian (of free motion): $h(\vec{r})=m-\frac{1}{2 m} \nabla_{\vec{r}}^{2}$. The two-pion wave function reads then [6]:

$$
\begin{aligned}
\psi_{\pi \pi}\left(\vec{r}_{a}, \vec{r}_{b} ; t\right)=\frac{\theta\left(t_{a}-t_{b}\right)}{\sqrt{2}} \quad & {\left[\psi_{a}\left(\vec{r}_{a} ; t_{a}\right) e^{-i h\left(\vec{r}_{b}\right)\left(t_{a}-t_{b}\right)} \psi_{b}\left(\vec{r}_{b} ; t_{b}\right)\right.} \\
& \left.+\psi_{a}\left(\vec{r}_{b} ; t_{a}\right) e^{-i h\left(\vec{r}_{a}\right)\left(t_{a}-t_{b}\right)} \psi_{b}\left(\vec{r}_{a} ; t_{b}\right)\right]
\end{aligned}
$$

The time evolution of the wave function(s) is described the most easily by introducing the Fourier decomposition into the momentum eigenstates. The two-particle amplitude corresponding to the appearance of the second $\pi$ meson at the time moment $t_{a}$ can be represented then as follows [6]:

$$
\begin{aligned}
A_{a b}^{(a)}\left(\vec{p}_{a}, \vec{p}_{b}\right)= & \sqrt{2} \theta\left(t_{a}-t_{b}\right) \int \frac{d^{3} Q}{(2 \pi)^{3}} \frac{d^{3} k}{(2 \pi)^{3}} d^{3} r d^{3} R e^{i(\vec{Q}-\vec{P}) \cdot \vec{R}} e^{i \Omega(\vec{Q}, \vec{k}) t_{a}} \\
& \times \cos \left(\frac{\vec{q} \vec{r}}{2}\right) \tilde{\psi}_{a}\left(\frac{\vec{Q}}{2}+\vec{k} ; t_{a}\right) \tilde{\psi}_{b}\left(\frac{\vec{Q}}{2}-\vec{k} ; t_{b}\right)
\end{aligned}
$$

Here $\tilde{\psi}(\vec{k} ; t)$ is the Fourier transform of the one-particle wave function defined as follows

$$
\psi(\vec{r} ; t)=\int \frac{d^{3} k}{(2 \pi)^{3}} \tilde{\psi}(\vec{k} ; t) e^{i(\vec{k} \vec{r}-\omega t)}
$$

and $\omega=\sqrt{m_{\pi}^{2}+\vec{k}^{2}}$. In eq.(13) $\vec{Q}=\vec{k}_{a}+\vec{k}_{b}$ and $\vec{k}=\left(\vec{k}_{a}-\vec{k}_{b}\right) / 2$. Finally, $\Omega(\vec{Q}, \vec{k}) \equiv E-\omega(\vec{Q} / 2+\vec{k})-\omega(\vec{Q} / 2-\vec{k})$. The integral over the c.m.s. coordinate $\vec{R}$ yields $(2 \pi)^{3} \delta^{(3)}(\vec{Q}-\vec{P})$, while the integration over $\vec{r}$ produces a Fourier image of the $\cos (\vec{q} \vec{r} / 2)$ function $\Phi(\vec{q} / 2, \vec{k})$ :

$$
\begin{aligned}
A_{a b}^{(a)}\left(\vec{p}_{a}, \vec{p}_{b}\right)= & \theta\left(t_{a}-t_{b}\right) \int \frac{d^{3} k}{(2 \pi)^{3}} e^{i \Omega(\vec{P} / 2, \vec{k}) t_{a}} \Phi(\vec{q} / 2, \vec{k}) \\
& \times \tilde{\psi}_{a}\left(\vec{P} / 2+\vec{k} ; t_{a}\right) \tilde{\psi}_{b}\left(\vec{P} / 2-\vec{k} ; t_{b}\right)
\end{aligned}
$$

An alternative situation when the particle in the state $a$ was emitted earlier at the time $t_{a}$ and the other particle appeared later at the time $t_{b}$ yields similar expression [6]:

$$
\begin{aligned}
A_{a b}^{(b)}\left(\vec{p}_{a}, \vec{p}_{b}\right)= & \theta\left(t_{b}-t_{a}\right) \int \frac{d^{3} k}{(2 \pi)^{3}} e^{i \Omega(\vec{P} / 2, \vec{k}) t_{b}} \Phi(\vec{q} / 2, \vec{k}) \\
& \times \tilde{\psi}_{a}\left(\vec{P} / 2+\vec{k} ; t_{a}\right) \tilde{\psi}_{b}\left(\vec{P} / 2-\vec{k} ; t_{b}\right)
\end{aligned}
$$


The total amplitude is the sum of two components (eq.(15) and (16) corresponding to two time orderings of the particles emission:

$$
A_{a b}\left(\vec{p}_{a}, \vec{p}_{b}\right)=A_{a b}^{(a)}\left(\vec{p}_{a}, \vec{p}_{b}\right)+A_{a b}^{(b)}\left(\vec{p}_{a}, \vec{p}_{b}\right)
$$

Using this amplitude we calculate the two-particle cross section which is defined as follows [6]

$$
P_{2}\left(\vec{p}_{a}, \vec{p}_{b}\right)=\sum_{a b, a^{\prime} b^{\prime}} \int d t_{a} d t_{b} d t_{a^{\prime}} d t_{b^{\prime}} \rho_{a b, a^{\prime} b^{\prime}} A_{a^{\prime} b^{\prime}}^{*}\left(\vec{p}_{a}, \vec{p}_{b}\right) A_{a b}\left(\vec{p}_{a}, \vec{p}_{b}\right)
$$

Here $\rho_{a b, a^{\prime} b^{\prime}}$ is the density matrix of the source which contains information on the probability distribution for the two-particle quantum numbers $(a, b)$ and for the emission times $\left(t_{a}, t_{b}\right)$.

Independent emission of two particles is ensured by a factorizable ansatz for the density matrix [6]:

$$
\rho_{a b, a^{\prime} b^{\prime}}=\nu_{a a^{\prime}} \rho\left(t_{a}, t_{a^{\prime}}\right) \nu_{b b^{\prime}} \rho\left(t_{b}, t_{b^{\prime}}\right)
$$

The probability of the two-particle emission consists of four terms:

$$
P_{2}\left(\vec{p}_{a}, \vec{p}_{b}\right)=P^{(a a)}+P^{(b b)}+P^{(a b)}+P^{(b a)}
$$

The reader is referred to 6 for the proof that $P^{(a b)}=P^{(b a)}=0$ and $P^{(a a)}=$ $P^{(b b)}$ so that

$$
P_{2}\left(\vec{p}_{a}, \vec{p}_{b}\right)=2 P^{(a a)}
$$

Following [6] we define the single particle density $S(X, K)$ of the source:

$$
\begin{aligned}
S(X, K)= & \int d^{4} x e^{i(K \cdot x)} \rho\left(X^{0}+x^{0} / 2, X^{0}-x^{0} / 2\right) \\
& \times \sum_{a a^{\prime}} \nu_{a a^{\prime}} \psi_{a}(X+x / 2) \psi_{a^{\prime}}^{*}(X-x / 2)
\end{aligned}
$$

where

$$
X^{0}=\frac{1}{2}\left(t_{a}+t_{a^{\prime}}\right), \quad x^{0}=t_{a}-t_{a^{\prime}}
$$

and the 4-momentum $K$ equals:

$$
\vec{K}=\frac{\vec{p}_{a}+\vec{p}_{b}}{2} ; \quad K_{0}=\frac{E_{a}+E_{b}}{2}
$$


Then the two-particle probability (18) can be expressed in terms of the singleparticle density [6]:

$$
\begin{aligned}
P_{2}\left(\vec{p}_{a}, \vec{p}_{b}\right)= & \int d^{4} X d^{4} Y[S(X, K+q / 2) S(Y, K-q / 2) \\
& \left.+e^{i q(X-Y)} S(X, K) S(Y, K)\right]
\end{aligned}
$$

Here $q_{0}=E_{a}-E_{b}$ and $\vec{q}=\vec{p}_{a}-\vec{p}_{b}$. Remind that the expression for the twoparticle probability was obtained in the frame where $\vec{p}_{a}+\vec{p}_{b} \approx 0$ implying that $q_{0} \approx 0$. Single particle spectrum is given by the expression:

$$
P_{1}(\vec{p})=\int d^{4} X S(X, p)
$$

with the 4-momentum $p=\left(\sqrt{\vec{p}^{2}+m_{\pi}^{2}}, \vec{p}\right)$

We are now in position to investigate the two-particle correlation function $C\left(\vec{p}_{a}, \vec{p}_{b}\right) \equiv C(\vec{q}, \vec{K}):$

$$
C(\vec{q}, \vec{K})=\frac{P_{2}\left(\vec{p}_{a}, \vec{p}_{b}\right)}{P_{1}\left(\vec{p}_{a}\right) P_{1}\left(\vec{p}_{b}\right)}
$$

We simplify this expression by separating the time and the spatial dependencies of the single-particle density $S(X, K)$ (eq.(22)). To this end we introduce a short-hand notation

$$
\rho(T, t) \equiv \rho\left(X^{0}+x^{0} / 2 ; X^{0}-x^{0} / 2\right)
$$

denoting the rest of the $S(X, K)$ as $\tilde{S}$ :

$$
\tilde{S}(\vec{R}, \vec{K})=\int d^{3} r e^{i \vec{K} \vec{r}} \sum_{a a^{\prime}} \nu_{a a^{\prime}} \psi_{a}(\vec{R}+\vec{r} / 2) \psi_{a^{\prime}}^{*}(\vec{R}-\vec{r} / 2)
$$

so that

$$
S(X, K)=\int d t e^{i E t} \rho(T, t) \tilde{S}(\vec{R}, \vec{K}) ; \quad X \equiv(T, \vec{R})
$$

Then the two-particle probability (25) can be cast in the form:

$$
\begin{aligned}
& P_{2}=\int d T_{1} d T_{2} d^{3} R_{1} d^{3} R_{2} \int d t_{1} d t_{2}\left\{e^{i E_{a} t_{1}} e^{i E_{b} t_{2}} \rho_{1} \rho_{2} \tilde{S}\left(\vec{R}_{1}, \vec{p}_{a}\right) \tilde{S}\left(\vec{R}_{2}, \vec{p}_{b}\right)\right. \\
& \left.+e^{i\left(E_{a}-E_{b}\right)\left(T_{1}-T_{2}\right)} e^{i \vec{q}\left(\vec{R}_{1}-\vec{R}_{2}\right)} e^{i K_{0} t_{1}} e^{i K_{0} t_{2}} \rho_{1} \rho_{2} \tilde{S}\left(\vec{R}_{1}, \vec{K}_{1}\right) \tilde{S}\left(\vec{R}_{2}, \vec{K}_{2}\right)\right\}
\end{aligned}
$$


where we introduced for notational brevity $\rho_{i} \equiv \rho\left(T_{i}, t_{i}\right) ; i=1,2$. Note that

in the considered frame $\vec{K} \approx 0$ the energies of the pions are almost equal to each other $\left(E_{a} \approx E_{b}\right)$, implying that $K_{0} \approx E_{a}\left(\approx E_{b}\right)$. The $P_{2}$ probability may be simplified further by introducing an (energy-dependent) factor

$$
\lambda(E)=\int d T d t \rho(T, t) e^{i E t}
$$

We arrive at

$$
\begin{aligned}
& P_{2}\left(\vec{p}_{a}, \vec{p}_{b}\right)=\lambda\left(E_{a}\right) \lambda\left(E_{b}\right) \int d^{3} R_{1} d^{3} R_{2} \\
& \left\{\tilde{S}\left(\vec{R}_{1}, \vec{p}_{a}\right) \tilde{S}\left(\vec{R}_{2}, \vec{p}_{b}\right)+e^{i\left(\vec{p}_{a}-\vec{p}_{b}\right)\left(\vec{R}_{1}-\vec{R}_{2}\right)} \tilde{S}\left(\vec{R}_{1}, \frac{\vec{p}_{a}+\vec{p}_{b}}{2}\right) \tilde{S}\left(\vec{R}_{2}, \frac{\vec{p}_{a}+\vec{p}_{b}}{2}\right)\right\} .
\end{aligned}
$$

The single-particle probability in these notations reads:

$$
P_{1}\left(\vec{p}_{a}\right)=\lambda\left(E_{a}\right) \int d^{3} R \tilde{S}\left(\vec{R}, \vec{p}_{a}\right)
$$

with the similar expression for $P_{1}\left(\vec{p}_{b}\right)$.

We obtain finally the two-particle correlation function:

$$
\begin{aligned}
& C\left(\vec{p}_{a}, \vec{p}_{b}\right)=1+ \\
& \int d^{3} R_{1} d^{3} R_{2} e^{\left(\vec{p}_{a}-\vec{p}_{b}\right)\left(\vec{R}_{1}-\vec{R}_{2}\right)} \tilde{S}\left(\vec{R}_{1}, \frac{\vec{p}_{a}+\vec{p}_{b}}{2}\right) \tilde{S}\left(\vec{R}_{2}, \frac{\vec{p}_{a}+\vec{p}_{b}}{2}\right) \\
& \left\{\int d^{3} R_{1} \tilde{S}\left(\vec{R}_{1}, \vec{p}_{a}\right) \int d^{3} R_{2} \tilde{S}\left(\vec{R}_{2}, \vec{p}_{b}\right)\right\}^{-1}
\end{aligned}
$$

Let us consider now a bound state of two baryons. As it was stated above, in the $N_{c} \rightarrow \infty$ limit they can be considered as heavy sources of $\pi$ mesons. Neglecting recoil due to the pion emission we can write the spatial distribution of the sources as

$$
\psi(\vec{r})=\gamma\left\{\delta\left(\vec{r}-\vec{R}_{\Delta \Delta} / 2\right)+\delta\left(\vec{r}+\vec{R}_{\Delta \Delta} / 2\right)\right\}
$$

where $\vec{R}_{\Delta \Delta}$ is the distance between two $\Delta$-isobars (baryons) and $\gamma$ is a normalization factor. (Hereafter for notational brevity we omit the wave function subscript denoting the charge state of the emitted pion). Inserting the wave function into the expression for the $\tilde{S}$ (eq.(29) ) we arrive at

$$
\tilde{S}(\vec{R}, \vec{p})=\gamma^{2}\left\{\delta\left(\vec{R}-\vec{R}_{\Delta \Delta} / 2\right)+\delta\left(\vec{R}+\vec{R}_{\Delta \Delta} / 2\right)\right\}
$$


and the corresponding single-particle distribution

$$
P_{1}\left(\vec{p}_{a}\right)=\lambda\left(E_{a}\right) 2 \gamma^{2}
$$

The two-particle distribution calculated with the same source function (37) reads

$$
P_{2}\left(\vec{p}_{a}, \vec{p}_{b}\right)=2 \gamma^{4}\left(1+\cos \left(\vec{q} \vec{R}_{\Delta \Delta}\right)\right)
$$

where $\vec{q}=\vec{p}_{a}-\vec{p}_{b}$. Note that in deducing $P_{2}$ only those configuration were taken into consideration when each heavy baryon emits a pion, i.e. the $\pi$ mesons are emitted in different spatial points.

Inserting the single- (eq.(38)) and two-particle (eq. (39)) distributions in the expression (27) we arrive at the correlation function for pions emitted by two heavy sources separated by the distance $\vec{R}$ (since it will not cause any confusion the subscript $\Delta \Delta$ is omitted hereafter):

$$
C_{\vec{R}}=1+\cos (\vec{q} \vec{R})
$$

Again, only those configurations were taken into account when each $\Delta$-isobar de-excitates by the emission of the $\pi$-meson. It implies that the $C_{\vec{R}}$ correlation function is contributed by the configurations when the emission points of the $\pi$-mesons are spatially separated. Note also that since $\vec{p}_{a}+\vec{p}_{b} \approx 0$, the relative momentum of two pions equals $\vec{q} \approx 2 \vec{p}_{a}$.

The correlation function (40) depends parametrically on the distance between pion-emitting sources $\vec{R}$. Two baryons being bound, their spatial distribution is governed by the bound-state wave function $\phi(\vec{R})$ : the probability that the baryons are at the distance $\vec{R}$ is given by the $|\phi(\vec{R})|^{2}$. The spatial distribution of pion-emitting sources does not affect the single-particle distribution (38), whereas the two-particle distribution (39) and, consequently, the two-pion correlation function $C_{\vec{R}}$ is modified. The observable correlation function

$$
C=\int d^{3} R C_{\vec{R}}|\phi(\vec{R})|^{2}
$$

reads

$$
C\left(\vec{p}_{a}, \vec{p}_{b}\right)=1+\int d^{3} R \cos (\vec{q} \vec{R})|\phi(\vec{R})|^{2}
$$

This expression shows that the two-pion correlation function with $\vec{q}$ varying "scans" the wave function of the baryon relative motion. 
Eq. (42) becomes more "transparent" in the case of an $S$-wave two-baryon bound state. Assuming a simplest deuteron-like wave function

$$
\phi(\vec{R})=\sqrt{\frac{\kappa}{2 \pi}} \frac{e^{-\kappa R}}{R}
$$

where $\kappa$ is the bound-state momentum $(\kappa=\sqrt{\varepsilon M}, \varepsilon$ is the binding energy and $M$ is the reduced mass of the pair of baryons) after straightforward integration over angular variables in eq. (42) we arrive at

$$
C=1+2 \kappa \int e^{-2 \kappa R} \frac{\sin (q R)}{q R} d R
$$

Introducing the $\chi=q / 2 \kappa$ ratio and calculating the integral we obtain the two-pion correlation function reading:

$$
C=1+\frac{\arctan \chi}{\chi}
$$

The dependence of the correlation function $C$ on $\chi$ is plotted in the figure. Let us consider it in more detail. Given a pair of bound $\Delta$-isobars they are localized in a domain with a spatial size $\bar{R} \sim 1 / \kappa$. For the values of $q$ small compared to $1 / \bar{R}$ (the ratio $\chi=q / 2 \kappa \ll 1$ ) both sources emit the $\pi$-mesons coherently resulting in the $100 \%$-interference and, hence, in $C \rightarrow 2$. In the opposite case $(q \gg 1 / \bar{R})$ because of the oscillation of the relative phase (see eq. (42)) the interference between the emitted pions is smeared out. It results in $C \rightarrow 1$ at large values of $\chi$. The devitation of the correlation function $C$ at large $\chi$ 's from the unity is proportional to the bound state momentum:

$$
C-1 \approx \frac{\pi}{2} \cdot \frac{2 \kappa}{q}
$$

In the case of unbound $\Delta$-isobars the wave function of their relative motion (squared) does not have the sense of the probability of finding two particles at some distance $\vec{R}$. (It is reflected, in particular, in the different normalization of the wave function of the continuous spectrum). This formal obstacle can be circumvented by considering a continuous-spectrum wave function as that of a bound state with vanishing binding energy. Then we get from the eq.(45) that $C=1$ for all values of $q$. This conclusion follows immediately from the qualitative consideration. Indeed, since in the 


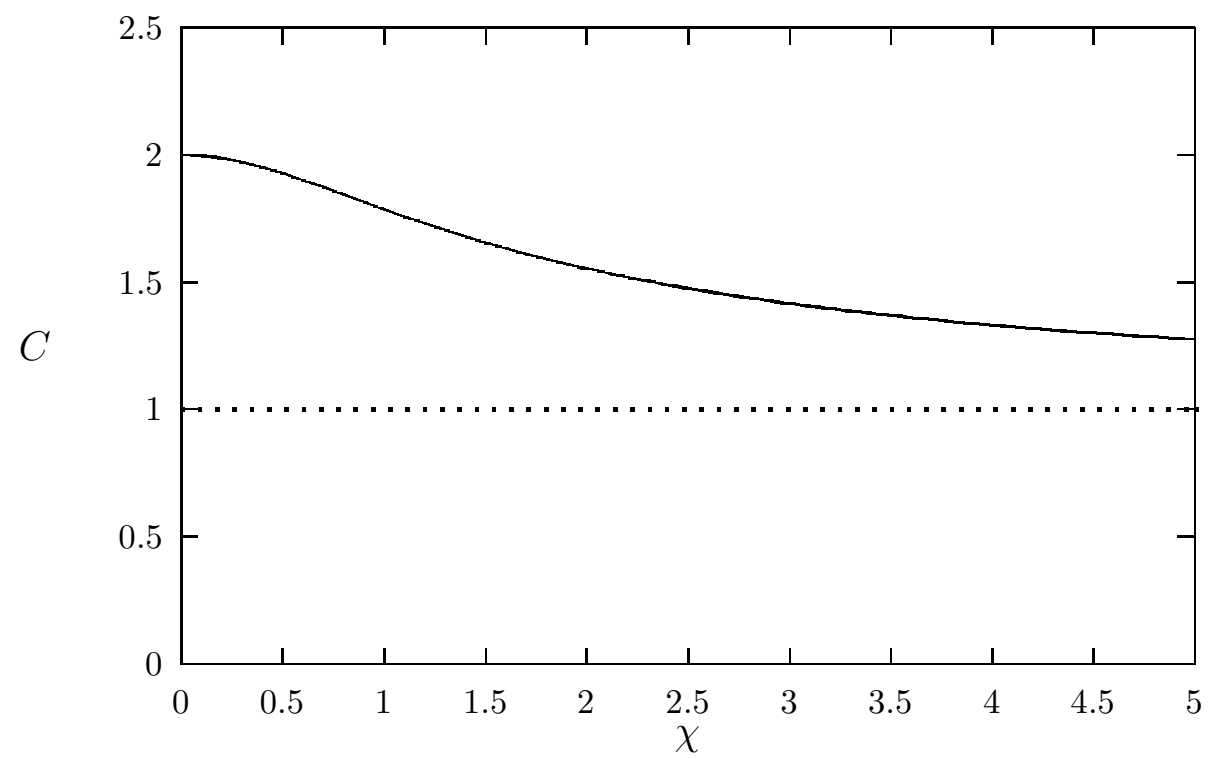

Figure 1: Momentum dependence of the correlation function in the presence (solid line) and absence (broken line) of a bound state

continuous spectrum arbitrarily large distances between emitting sources are possible the relative phase controlled by the $\vec{q} \vec{R}$ product will be large for any (nonvanishing) value of $q$. Inspection of (42) shows that because of rapid oscillations of the integrand $C=1$ for any unbound state.

When deducing correlation function (42) several approximations have been made. The first approximation is that the emission of a $\pi$-meson changes the discrete numbers of a baryon $B$ only (e.g. $\Delta \rightarrow N$ ) and does not affect its position. Such an assumption seems to be justified in the $N_{c} \rightarrow \infty$ limit where baryons may be considered as (infinitely) heavy particles. Before proceeding to the discussion of other approximations made we consider in more detail (possible) bound states. The very concept of a bound state of two baryons $B_{1}$ and $B_{2}$ assumes that they do not lose their identity. It can be translated in the requirement that the characteristic distance $\bar{R}$ between two particles exceeds their doubled size $b: \bar{R} \gtrsim 2 b$. Taking as a benchmark of the baryon's size that of the nucleon $(b \approx 0.5 \mathrm{fm})$ we arrive at the conclusion that the spatial dimensions of a two-baryon bound state are to be large: $\bar{R} \gtrsim 1 \mathrm{fm}$. The latter inequality translates into the limitation on the 
bound-state momentum $\kappa \approx 1 / \bar{R} \lesssim 200 \mathrm{MeV}$. The correlation function $C$ noticeably deviates from 1 for the values of $q \approx \kappa$ (see the figure). Strong correlation in the $\pi-\pi$ system are known to occur at the effective mass value $\approx 500 \div 600 \mathrm{MeV}$, i.e. at the values of $q$ in the same momentum range. One can conclude that in the $q$ range of interest $(q \approx 200 \mathrm{MeV})$ the correlations between pions are weak thus justifying the approximation made.

One more implicit assumption made consists in neglecting the $\pi$-meson rescattering on the (heavy) particles. Such rescattering could generate some phase of the pion wave function (11). The magnitude of the rescattering effect can be roughly estimated as follows. Let $\sigma$ be a cross section of the emitted $\pi$-meson rescattering on the other baryon. The probability of the process is controlled by the ratio $\sigma /\left(4 \pi \bar{R}^{2}\right)$. In the considered momentum range $|\vec{p}|=|\vec{q}| / 2 \approx 100 \div 200 \mathrm{MeV}$ the cross section of the $\pi N$ scattering

$\sigma_{\pi N} \approx 50 \div 100$ mbarn [7]. Then the rescattering parameter for $\bar{R} \gtrsim 2 \mathrm{fm}$ proves to be small $\lesssim 25 \%$.

Summarizing, possible shallow bound states of two baryons can be investigated by means of the pion interferometry. The momentum dependence of the two-particle correlation function in the "back-to-back" kinematics is controlled by the binding-energy value of two baryons. The latter can be determined experimentally from the slope of the two-particle correlation function at (small) momenta of the $\pi$-mesons. The proposed method suits best for the search of possible (loosely) bound states of two resonances which binding, because of the large width, can hardly be observed as a shift of a peak in corresponding effective-mass distributions.

The author is grateful to M.Polyakov and O.Patarakin for helpful discussions.

\section{References}

[1] R.Bilger et al, Phys.Lett. B269 (1991) 247

[2] H.Garcilazo, F.Fernández, A.Valcarce and R.D.Mota, Phys.Rev. C56 (1997) 84 
[3] U.Heinz, In: Correlations and Clustering Phenomena in Subatomic Physics, ed. by M.N.Harakeh et al., NATO Series B359, p. 137 (Plenum, NY, 1997)

[4] A.Manohar, Phys.Lett. B336 (1994) 502

[5] L.I.Schiff, Quantum Mechanics, Gustorff 1969

[6] D.Anchishkin, U.Heinz and P.Renk, nucl-th/9710051

[7] Particle Data Group, Phys.Rev. D54, Part I (1996) 1 